\title{
A Hospital-based Study of Renal Function Tests in Chronic Alcoholics
}

\author{
${ }^{1}$ Velu Malarkodi, ${ }^{2}$ Mala Malathi
}

\section{ABSTRACT}

Aim: The purpose of the study is to assess the glomerular filtration rate (GFR) using Modification of Diet in Renal Disease (MDRD) formulae, and to estimate renal parameters including electrolytes in chronic alcoholics and compare all the parameters with normal controls.

Materials and methods: A total of 50 male alcoholics were taken for the study on admission to the deaddiction center in Father Muller Medical College and Hospital. All the serum parameters were analyzed in auto analyzer Cobas c 311, and qualitative analysis of urine was done using dipsticks. Statistical evaluation was done using Student's t-test and Karl Pearson's correlation coefficient.

Results: The mean estimated GFR (eGFR) in alcoholics of our study was slightly increased with an increase in $32 \%$ of alcoholics. A significant negative correlation of eGFR with age $(p=0.027)$ and urea $(p=0.039)$ was seen. There was a significant decrease in the levels of urea $(p=-0.022)$ and potassium $(p=-0.008)$. There was a mean increase in uric acid and decrease in phosphate levels. Qualitative analysis of urine showed proteinuria and hematuria in alcoholics with eGFR $<110$.

Conclusion: The study showed a spectrum of variation in the renal parameters assayed, with a significant decrease in urea and potassium levels. Validation of the findings by large-scale cohort studies is needed.

Clinical significance: There is a significant decrease in the renal function with variations in electrolytes and a decrease in phosphate levels. Some cases also had proteinuria and hematuria, indicating progress toward chronic renal disease.

Keywords: Chronic alcoholism, Chronic renal disease, Estimated glomerular filtration rate, Modification of diet in renal disease.

How to cite this article: Malarkodi V, Malathi M. A Hospitalbased Study of Renal Function Tests in Chronic Alcoholics. Indian J Med Biochem 2018;22(1):22-25.

Source of support: Nil

Conflict of interest: None

${ }^{1}$ Assistant Professor, ${ }^{2}$ Professor and Head

${ }^{1}$ Department of Biochemistry, Government Sivagangai Medical College, Sivagangai, Tamil Nadu, India

${ }^{2}$ Department of Biochemistry, Father Muller Medical College Mangaluru, Karnataka, India

Corresponding Author: Velu Malarkodi, Assistant Professor Department of Biochemistry, Government Sivagangai Medical College, Sivagangai, Tamil Nadu, India, Phone: +917358558458 e-mail: drmalarkodi@gmail.com

\section{INTRODUCTION}

Alcohol ingestion is associated with a myriad of deleterious effects on the kidney, ranging from tubular dysfunction and various forms of acute renal failure. ${ }^{1}$ The World Health Organization statistics say that $4.5 \%$ of the global burden of disease and injury was attributable to alcohol. Worldwide, about $11.5 \%$ of drinkers have weekly heavy episodic drinking occasions. ${ }^{2}$ About 5.11 million consumers in Karnataka state annually consume 100.87 million liters of absolute alcohol equivalent. Per capita consumption of alcohol in Karnataka is about $2.98 \mathrm{~L}$ of absolute alcohol per year. ${ }^{3}$ Studies ${ }^{4-6}$ have linked higher serum creatinine and an increased risk of end-stage renal disease (ESRD) to higher alcohol intake. Based on the very few population-based studies conducted in India, the estimated prevalence of overt chronic kidney disease (CKD) ESRD is 0.79 to $1.39 \% .^{7}$ The relationship between alcohol consumption and CKD has been the subject of relatively little research. The GFR is a better indicator of renal disease than serum creatinine. A study found that men who consumed at least seven drinks weekly had an odds ratio of 0.71 to develop new onset of renal dysfunction. ${ }^{8}$ The present study intends to highlight the effect of chronic alcoholism on the renal function well before a rise in serum creatinine is seen.

In this study, we aim to assess eGFR using MDRD formulae along with the estimation of urea uric acid and electrolytes in serum of alcoholics and compare them with those of nonalcoholics. We perform qualitative urine analysis of alcoholics to detect abnormalities.

\section{MATERIALS AND METHODS}

A total of 50 male alcoholics aged 21 to 60 years were taken for the study, in comparison with 50 male nonalcoholic healthy controls, on admission to the deaddiction center in Father Muller Medical College and Hospital after institutional ethical committee clearance. Alcohol dependence was assessed by Alcohol Use Disorders Identification test (AUDIT) questionnaire. The AUDIT score above 8 indicated hazardous drinking and more than 15 in males indicates alcohol dependence. Harmful levels of absolute alcohol for males are $>50$ units $(400 \mathrm{gm}) /$ week. ${ }^{9}$ Alcoholdependent males with AUDIT score more than 15 were considered for this study after taking informed written 
A Hospital-based Study of Renal Function Tests in Chronic Alcoholics

Table 1: Analysis of parameters in alcoholics and controls

\begin{tabular}{|c|c|c|c|c|}
\hline Parameter & Alcoholics $(n=50)$ & Controls $(n=50)$ & Comparison & Significance \\
\hline Age & $40.2 \pm 9.82$ & $42.7 \pm 11.23$ & $p=0.239$ & NS \\
\hline eGFR & $111.03 \pm 23.99$ & $108.42 \pm 22.01$ & $p=0.572$ & NS \\
\hline Urea & $20.14 \pm 9.78$ & $24.16 \pm 7.24$ & $p=0.022$ & sig \\
\hline Creatinine & $0.85 \pm 0.17$ & $0.85 \pm 0.14$ & $p=0.948$ & NS \\
\hline Uric acid & $5.63 \pm 1.22$ & $5.18 \pm 1.29$ & $p=0.076$ & NS \\
\hline Sodium & $138.94 \pm 4.5$ & $137 \pm 4.5$ & $p=0.068$ & NS \\
\hline Potassium & $3.8 \pm 0.49$ & $4 \pm 0.5$ & $p=0.008$ & $\mathrm{HS}$ \\
\hline Chloride & $100.6 \pm 5.2$ & $99.6 \pm 4.9$ & $p=1.034$ & NS \\
\hline Bicarbonate & $23.02 \pm 3.21$ & $23.4 \pm 2.3$ & $p=0.504$ & NS \\
\hline Phosphate & $3.72 \pm 1.02$ & $4.01 \pm .61$ & $P=0.088$ & NS \\
\hline
\end{tabular}

NS: Not significant; sig: Significant correlation with $p<0.05$; HS: Highly significant correlation with $p<0.01$

consent. Alcoholics with other associated premorbid conditions like diabetes mellitus, advanced liver disease, and those on prolonged medication known to affect the renal function were excluded. Serum parameters were analyzed in autoanalyzer Cobas c 311. The eGFR was calculated using the MDRD formula based on serum creatinine levels. According to CKD National Kidney Foundation The Kidney Disease Outcome Quality Improvement (NKF K/DOQI), the MDRD formula ${ }^{10}$ is recommended for eGFR calculation.

$\mathrm{MDRD}=186 \times(\mathrm{SCr})^{1.154} \times[\text { age }(\text { years })]^{0.203} \times(0.742$ if female $) \times(1.210$ if African-American), where $\mathrm{SCr}$ is serum creatinine in $\mathrm{mg} / \mathrm{dL}$

The qualitative analysis of the urine was done using DX urine test 10 reagent strips from Piramal health care Limited. Statistical analysis by Statistical Package for the Social Sciences software was done using student t-test and Karl Pearson's correlation coefficient.

\section{RESULTS}

Mean eGFR in alcoholics was increased in alcoholics compared with normal. Student's t-test was done to compare the parameters of alcoholics with controls (Table 1). A
Table 2: Correlation of eGFR with the other parameters

\begin{tabular}{lcllll}
\hline & \multicolumn{2}{c}{ Alcoholics } & & \multicolumn{2}{c}{ Controls } \\
\cline { 2 - 3 } \cline { 5 - 6 } Parameters & Correlation & $p$-value & & Correlation & $p$-value \\
\hline Age & $-0.312^{\mathrm{a}}$ & 0.027 & & -0.262 & 0.067 \\
Urea & $-0.293^{\mathrm{a}}$ & 0.039 & & -0.255 & 0.074 \\
Creatinine & $-0.916^{\mathrm{b}}$ & 0 & & $-0.929^{\mathrm{b}}$ & 0 \\
Uric acid & -0.085 & 0.557 & & $-0.337^{\mathrm{a}}$ & 0.017 \\
Sodium & 0.187 & 0.193 & & 0.130 & 0.368 \\
Potassium & -0.079 & 0.587 & & $-0.379^{\mathrm{b}}$ & 0.007 \\
Chloride & 0.111 & 0.441 & & 0.110 & 0.445 \\
Bicarbonate & 0.026 & 0.857 & & -0.149 & 0.302 \\
Phosphate & 0.008 & 0.954 & & -0.140 & 0.332 \\
\hline
\end{tabular}

${ }^{\mathrm{a}}$ Correlation is significant at the 0.05 level (2-tailed); ${ }^{\mathrm{b}}$ Correlation is significant at the 0.01 level (2-tailed)

significant decrease in the urea and potassium levels was seen. There was a mean increase in uric acid, sodium and chloride. A mean decrease in the phosphate levels was also noted (Table 2). Karl Pearson's correlation of eGFR with other parameters showed a significant correlation with age, urea, uric acid, and potassium (Table 2). The eGFR was divided into quartiles based on mean and standard deviation and the changes in urea are noted (Graph 1), and a significant decrease in potassium in alcoholics is seen (Graph 2).

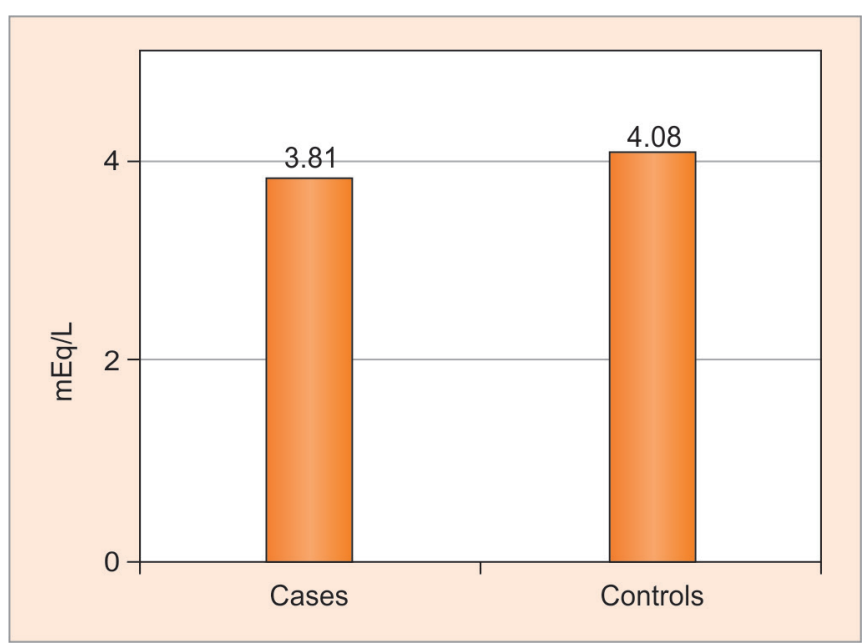

Graph 2: Serum potassium levels in cases and controls

Graph 1: Urea levels in eGFR quartiles of alcoholics 


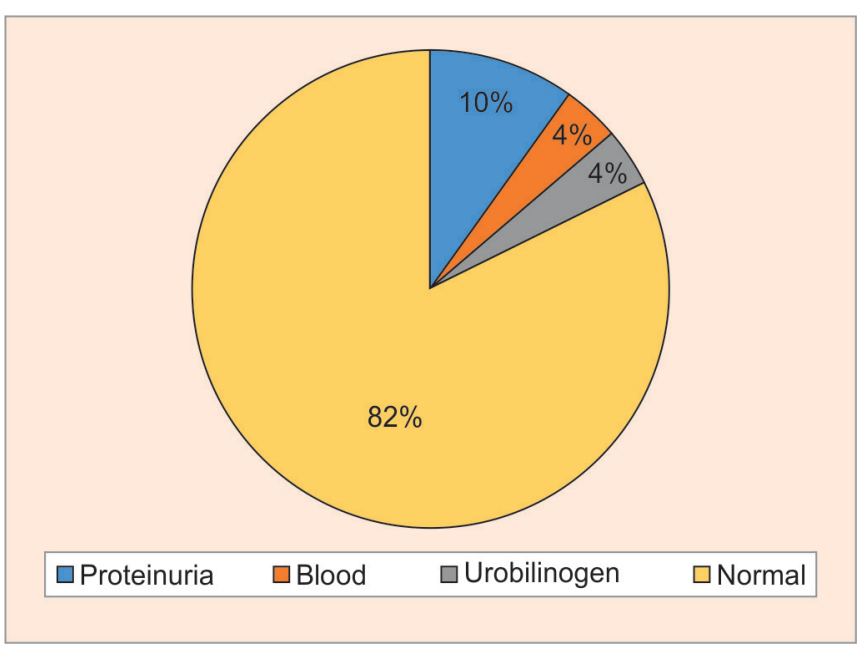

Graph 3: Incidence of abnormal constituents of urine in alcoholic subjects

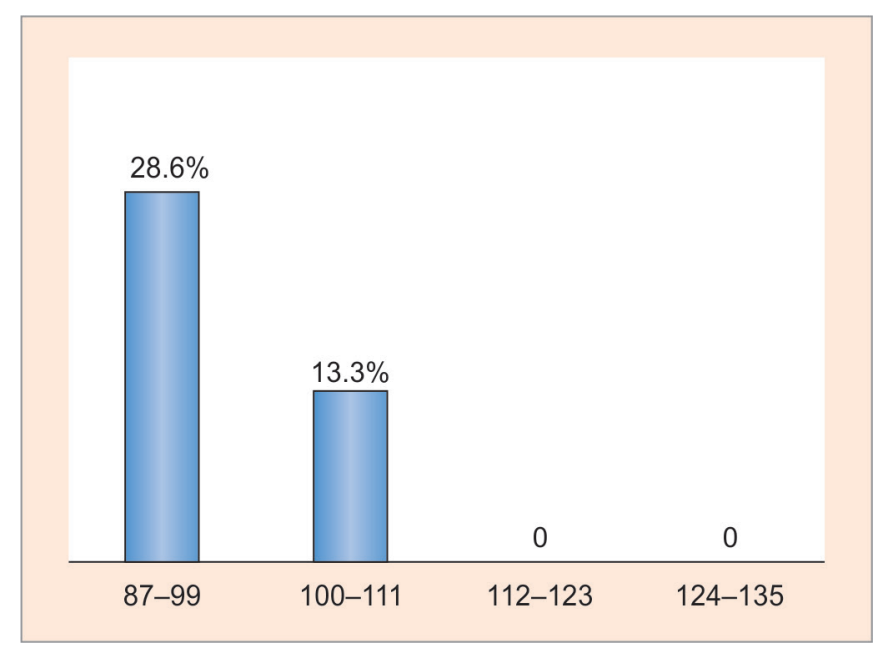

Graph 5: Incidence of proteinuria in percentage with respect to eGFR quartiles

The analysis of urine revealed that $49 \%$ of the subjects had diuresis; $51 \%$ of alcoholics had acidification of the urine. Some of the subjects had abnormal findings in the urine like proteinuria $(10 \%)$, hematuria $(4 \%)$, and urobilinogen (4\%) in the study population (Graphs 3 and 4). The eGFR range was divided into quartiles and the result of urine examination was analyzed. The incidence of proteinuria was high in the first quartile of eGFR $(28.6 \%)$ and was nil in the third and fourth quartiles (Graph 5).

\section{DISCUSSION}

Alcohol consumption may affect the regulation of vasoactive substances, consequently affecting renal hemodynamics and GFR. ${ }^{11}$ Estimating GFR using the formulae based on serum creatinine was equally accurate when compared with the clearance studies according to the NKF K/DOQI guidelines. ${ }^{10,12}$ The simplified MDRD equation is widely used in daily clinical practice and its accuracy has been studied in normorenal and CKD

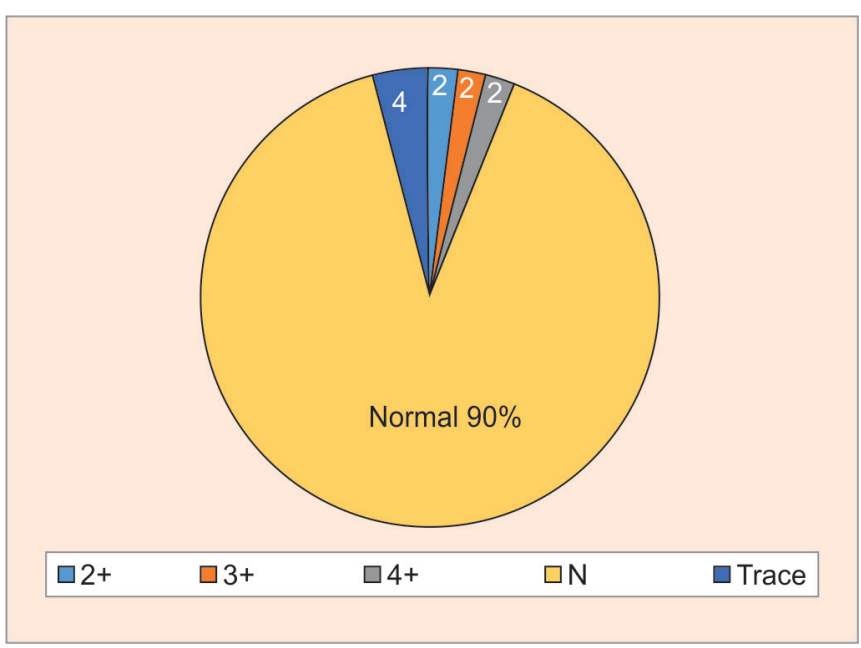

Graph 4: Incidence of proteinuria in the alcoholic subject

patients. ${ }^{10}$ Subjects with chronic alcohol consumption have significantly higher estimated GFR and creatinine clearance values than nondrinkers. ${ }^{11}$ Males consuming seven or more units of alcohol per week were associated with a significant increase in eGFR over 7 years of follow-up versus abstinence, although the same was not observed for females. ${ }^{13}$ Our study showed a mean increase in eGFR. Similar to our study, decreased urea was reported in two studies. ${ }^{14,15}$ An animal study ${ }^{16}$ on rat kidneys has shown that the urea and creatinine levels have been elevated over the weeks of ethanol exposure. A case-control study ${ }^{6}$ concluded that individuals who consumed 2 or fewer drinks per day had higher serum creatinine concentrations than matched controls. This study found an increase in uric acid levels. The clearance of uric acid is hampered and there is also increased synthesis in alcoholics. ${ }^{17-20}$ The values obtained for sodium and potassium in a study done by Oduola et $\mathrm{al}^{21}$ were significantly lower in heavy drinkers. The abnormalities in the electrolytes are also supported by the study done by Mahboob et al, and a study by Sergio De Marchi et $\mathrm{al}^{22}$ showed that $13 \%$ had hypokalemia. This finding of hypokalemia is also supported by many studies. ${ }^{17,23}$ Our study has shown a mean decrease in the phosphate levels. A study by De Marchi et $\mathrm{al}^{22}$ also showed that $30 \%$ had hypophosphatemia. About $18 \%$ of the present study population had hyperphosphatemia. A study by Martin et $\mathrm{al}^{23}$ showed that there were $13.6 \%$ of alcoholics with hyperphosphatemia and hypophosphatemia each. These findings are also supported by the study done by Liamis et al. ${ }^{17}$ There was diuresis in most of the alcoholics (49\%) in our study which coincides with the finding in many studies. ${ }^{24,25}$ Proteinuria, hematuria, and leukocyturia in chronic alcoholics were mostly pronounced in abstinence, particularly in those with a long-term alcoholism. ${ }^{26}$ 


\section{CONCLUSION}

In conclusion, the study showed that the mean eGFR was higher, indicating predominant diuresis. The study showed a spectrum of variation in the renal parameters assayed, with a significant decrease in urea and potassium. The qualitative analysis of urine showed proteinuria and hematuria, which indicates renal impairment. Validation of the findings by large-scale cohort studies is needed. The renal dysfunction in alcoholics is to be ascertained using newer markers like Cystatin C in Indian population.

\section{CLINICAL SIGNIFICANCE}

Alcohol has widespread effect on various tissues; in this study, there is a significant decrease in the renal function with variations in electrolytes and a decrease in the phosphate levels. Some cases also had proteinuria and hematuria, indicating the progress toward chronic renal disease. Hence, chronic alcoholism has a deleterious effect on the renal function as well as other organs due to the electrolyte imbalance and reduced GFR. It also could be assumed from this study that the renal tubules are also affected other than glomerular function.

\section{REFERENCES}

1. Adewale A, Onyekachilfudu. Kidney injury, fluid, electrolyte and acid-base abnormalities in alcoholics. Niger Med J 2014 Mar-Apr;55(2):93-98.

2. Benegal V. India: alcohol and public health. Addiction 2005 Aug;100(8):1051-1056.

3. Benegal V, Gururaj G, Murthy P. Report on a WHO collaborative project on unrecorded consumption of alcohol in Karnataka, India. Bangalore, India: National Institute of Mental Health and Neurosciences; 2003.

4. Knight EL, Meir JS, Rimm EB, Hankinson SE, Curhan GC. Moderate alcohol intake and renal function decline in women prospective study. Nephrol Dial Transplant 2003 Aug;18(8):1549-1554.

5. Van Thiel DH, Williams WD, Gavaler JS, Little JM, Estes LW, Rabin BS. Ethanol-its nephrotoxic effect in the rat. Am J Pathol 1977 Oct;89(1):67-84.

6. Savdie E, Grosslight GM, Adena MA. Relation of alcohol and cigarette consumption to blood pressure and serum creatinine levels. J Chronic Dis 1984;37:617-623.

7. Varma PP, Raman DK, Ramakrishnan TS, Singh P, Verma A. Prevalence of early stages of chronic kidney diseases in apparently healthy central government employees in India. Nephrol Dial Transpl 2010 Mar;25(9):3017-3025.

8. Schaeffner ES, Kurth T, de Jong PE. Alcohol consumption and the risk of renal dysfunction in apparently healthy men. Arch Intern Med 2005 May;165(9):1048-1053.
9. Sharpe PC. Biochemical detection and monitoring of alcohol abuse and abstinence. Ann Clin Biochem 2001 Nov;38(Pt 6): 652-664.

10. Tidman M, Sjostrom P, Jones I. A comparison of GFR estimating formulae based upon s-cystatin $\mathrm{C}$ and s-creatinine and a combination of the two. Nephrol Dial Transplant 2008 Jan;23(1):154-160.

11. Chung FM, Yang YH, Shieh TY, Shin SJ, Tsai JC, Lee YJ. Effect of alcohol consumption on estimated glomerular filtration rate and creatinine clearance rate. Nephrol Dial Transplant 2005 Aug;20(8):1610-1616.

12. Hillbom M, Saloheimo P, Juvela S. Alcohol consumption, blood pressure, and the risk of stroke. Curr Hypertens Rep 2011 Jun;13(3):208-213.

13. Kronborg J, Solbu M, Njølstad I, Toft I, Eriksen BO. Predictors of change in estimated GFR: a population-based 7-year follow-up from the Tromso study. Nephrol Dial Transplant 2008 Sep;23(9):2818-2826.

14. Holmuhamedov EL, Czerny C, Beeson CC, Lemasters JJ. Ethanol suppresses ureagenesis in Rat hepatocytes: role of acetaldehyde. J Biol Chem 2012 Jan;287:7692-7700.

15. De Oliveira EP, Burini RC. High plasma uric acid concentration: causes and consequences. Diabetol Metab Syndr 2012 Apr;4(12):1-7.

16. Das SK, Varadhan S, Dhanya L, Mukherjee S, Vasudevan DM. Effects of chronic ethanol exposure on renal function tests and oxidative stress in kidney. Indian J Clin Biochem 2008 Oct;23(4):341-344.

17. Liamis GL, Milionis HJ, Rizos EC, Siamopoulos KC, Elisaf MS. Mechanisms of hyponatremia in alcoholics. Alcohol Alcohol 2000 Nov-Dec;35(6):612-616.

18. Lieber CS, Davidson CS. Some metabolic effects of ethyl alcohol. Am J Med 1962;32:319-327.

19. Sanghvi SR, Kellerman PS, Nanovic L. Beer potomania: an unusual cause of hyponatremia at high risk of complications from rapid correction. Am J Kidney Dis 2007;50(4):673-680.

20. Whitfield JB. Alcohol-related biochemical changes in heavy drinkers. Aust NZ J Med 1981;11:132-139.

21. Oduola T, Adeosun OG, Oduola TA, Agbaje NR, Raheem ZA. Drinking patterns: biochemical and haematological findings in alcohol consumers in Ile-Ife, Nigeria. Afr J Biotechnol 2005 Nov;4(11):1304-1308.

22. De MarchiS, Cecchin E, Basile A, Bertotti A, Nardini R, Bartoli E. Renal tubular dysfunction in chronic alcohol abuse-effects of abstinence. New Engl J Med 1993 Dec;239(26):1927-1935.

23. Martin HE, Mccuskey C, Tupikova N. Electrolyte disturbance in acute alcoholism - with particular reference to magnesium. Am J Clin Nutr 1959 Mar-Apr;7(2):191-196.

24. Ogata M, Mendelson JH, Mello NK. Electrolytes and osmolality in alcoholics during experimentally induced intoxication. Psychomatic Med 1968 Sep;30(5):463-488.

25. Udokang NE, Akpogomeh BA. Effect of volume loading with water, normal saline, palm wine and Lipton tea on urinary output, $\mathrm{pH}$, specific gravity, sodium and potassium concentrations in human subjects. Niger J Physiol Sci 2010 Jun-Dec;20(1-2):101-106.

26. Schaeffner E, Ritz E. Alcohol and kidney damage: a Janus faced relationship. Kidney Int 2012 Feb:81(9):816-818. 